\title{
Electromagnetically excited resonator sensors for remote mass detection and liquid phase sensing
}

\author{
Frieder Lucklum* and Bernhard Jakoby \\ Institute for Microelectronics, Johannes Kepler University Linz, A-4040 Linz, Austria \\ *E-mail: frieder.lucklum@jku.at
}

\begin{abstract}
Electromagnetic excitation of acoustic resonator sensors is a novel method with distinct advantages over piezoelectric resonators. A much wider range of transducer materials can be utilized, and remote excitation of resonant vibrations in a wide variety of different mode shapes is possible. These resonant modes display similar quality factors and characteristics as piezoelectric transducers and can be applied for mass detection and liquid phase sensing.
\end{abstract}

Key words: Electromagnetic excitation, EMAT, microacoustic sensor, face shear mode resonator.

\section{A. Introduction}

In recent years, microacoustic sensors have started to employ a wider variety of ultrasonic transducers. Besides the traditional piezoelectric quartz resonators, microfabrication technology has allowed to develop new excitation mechanisms, such as the piezoelectric film bulk acoustic wave resonator (FBAR) and the capacitive micromachined ultrasonic transducer (CMUT). A third transducer mechanism based on electromagnetic excitation and detection of acoustic waves has been known as the electromagnetic-acoustic transducer (EMAT) principle for several decades. Miniaturizing the excitation setup and applying it to micromachined resonator devices allows us to employ this technology as a microacoustic sensor as well [1-5].

Our work has focused on the study of suitable resonator materials, appropriate excitation and detection setups, as well as the characterization of these sensors for different applications in mass detection and liquid phase sensing. The classic EMAT advantages of remote excitation and detection of acoustic waves remain, while microfabrication technology allows for the utilization of a wide variety of resonator materials, ranging from pure metal samples, coated polymer films to silicon or glass elements. Furthermore, it is possible to excite different modes of vibration with the same or a similar setup. Flexural plate modes, face shear modes, and thickness shear modes can be used for sensing different properties.

In this contribution, we present the most recent work on design and simulation of the setup and the most suitable modes of vibration, as well as some measurement results to evaluate and verify our conclusions.

\section{B. Design and simulation results}

\section{B.1. Theoretical Considerations}

For the excitation of these vibration modes, eddy current generation is utilized. The eddy currents are induced in the conductive substrate or a conductive layer of the resonator element, e.g., by a planar coil. A superposed static magnetic field generates Lorentz forces that put the element into vibration. The resonator vibrating in the magnetic field induces a secondary voltage in the primary coil, which can be measured in the impedance of the coil. The impedance behavior is therefore related to the resonant mechanical behavior. The resonances, which are successfully excited by the specific setup, can thus be measured and tracked for sensing purposes.

The mechanical resonator can be modeled as an electrical series resonance circuit. This circuit is electromechanically coupled with the eddy currents, which can be modeled as a transformer coupling. The primary coil results in a mutual inductance with the induced eddy currents, therefore a complete electrically equivalent circuit has been developed for this type of transducer [7].

\section{B.2. Design Challenges}

Fig. 1 shows the basic measurement setup. With a vertically perpendicular static magnetic field, a planar

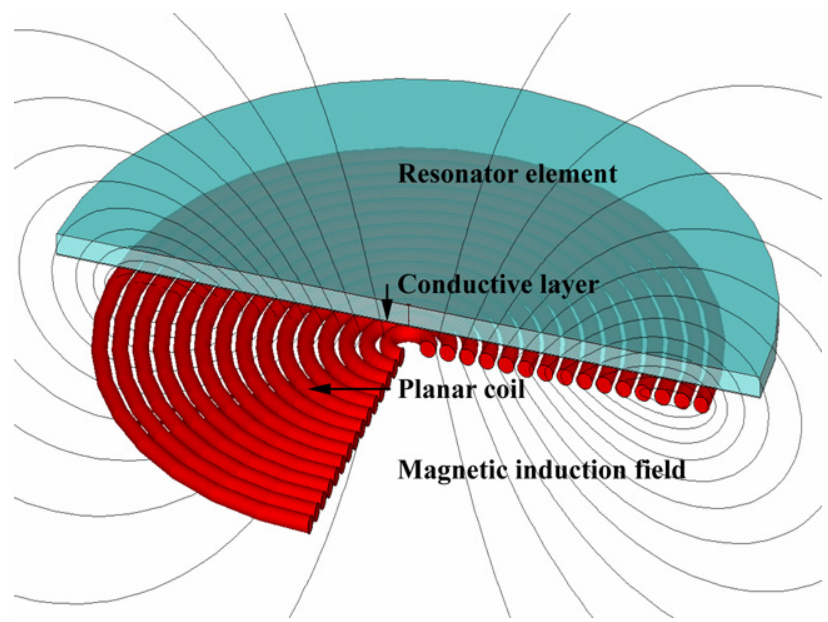

Fig. 1. Model of the excitation and detection setup with current in primary coil inducing eddy currents in the resonator. 


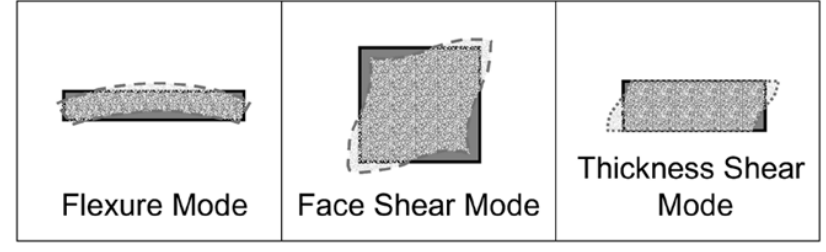

Fig. 2. Possible modes of vibration for membrane type transducer elements.

coil will always generate in-plane Lorentz forces, which favors the excitation of shear modes. Changing the magnetic flux to a lateral distribution will generate outof-plane forces, suitable for flexural plate modes (Fig. 2).

The second aspect of designing a suitable excitation setup is the actual mode shape. Shear modes in both isotropic and anisotropic elastic plates show a fundamental distribution of diagonal, circular, or radial nature. Higher harmonics and combinations of these fundamental modes can be utilized as well. A similar distinction can be made for flexural plate modes.

In order to excite these different mode shapes, a suitable Lorentz force distribution is necessary. The force distribution is directly dependent on both the magnetic field direction as described above and the flow direction of the eddy currents for planar transducer surfaces. The currents are primarily a mirror image of the excitation current flow. Therefore, different coil layouts, as circular and quadratic spiral coils, will excite different modes of vibration (Fig. 3). Additionally, we have proposed and shown the application of alternated magnetic fields, e.g., with two discrete permanent magnets attached to each other side by side, to enhance desired and eliminate unwanted force components [8].

Further design considerations include the application of an excitation layout and a detection coil. In that case, the excitation layout does not require a coil-like element. We have also designed and fabricated linear, meandrous and toroidal conductors for this purpose and are currently working on a suitable measurement setup.

\section{B.3. FEM simulation}

To investigate the different resonator elements and to find and examine suitable eigenmodes, we have utilized the FEM software COMSOL Multiphysics 3.3.
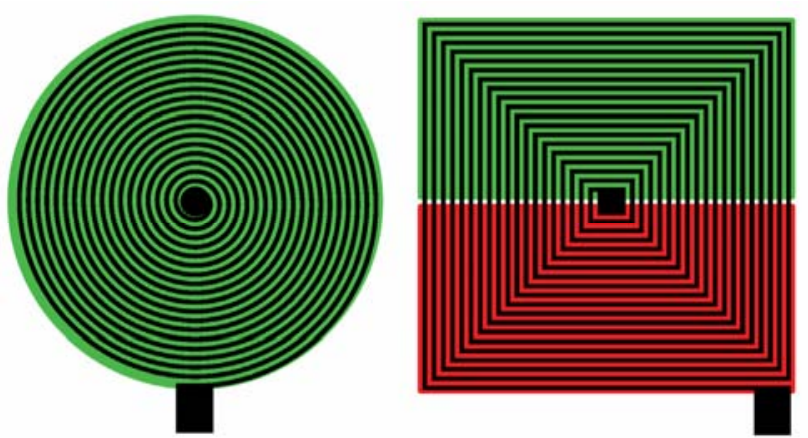

Fig. 3. Two coil designs and magnet placement for radial (left) and diagonal (right) force distribution.

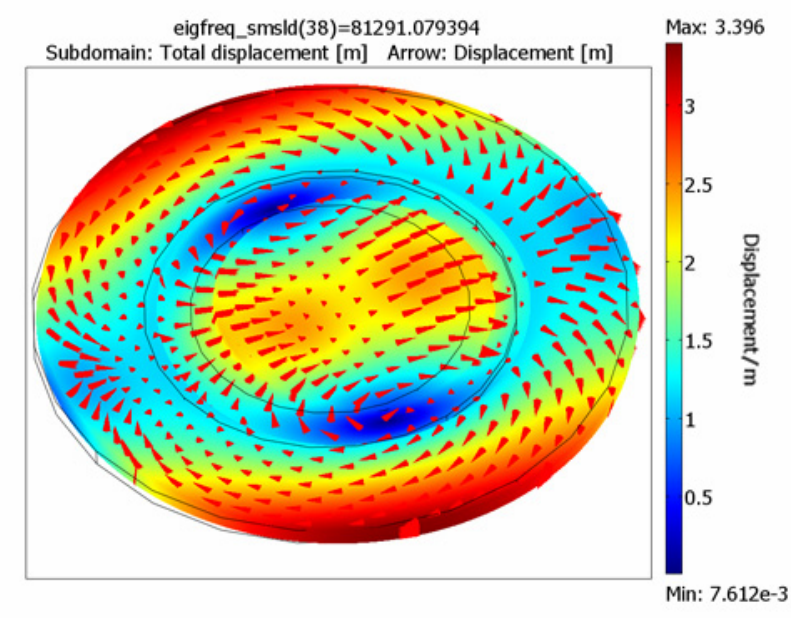

Fig. 4. Fundamental diagonal face shear mode at $81 \mathrm{kHz}$ for aluminum resonator with mesa structure.

This software is also being used to model the excitation mechanism accurately.

We focus on the FEM results for a round aluminum resonator with an active circular mesa structure of $15 \mathrm{~mm}$ diameter. This element was cast to form the energy trapping mesa structure in the center and dimensioned to fit the existing planar coils. As seen in Fig. 4, the first diagonal face shear mode appears at $81 \mathrm{kHz}$ for this element. However, the primary shear mode is superposed by a slight out-of-plane movement, induced by the element geometry. This out of plane movement is also evident in the experimental results, where we get additional damping in liquid environments due to compressional waves being generated by the out-of-plane movement. The 2nd harmonic of this diagonal face shear mode can be found at $171 \mathrm{kHz}$ and can also be detected in actual measurements. A slight vertical component in the active area is also evident here (Fig. 5), resulting in a similar resonant behavior.

For our liquid phase experiments, we have utilized two different modes of vibration, the circular face shear mode and a radial flexural plate mode (Fig. 6). Both modes are very different in the behavior in a fluid

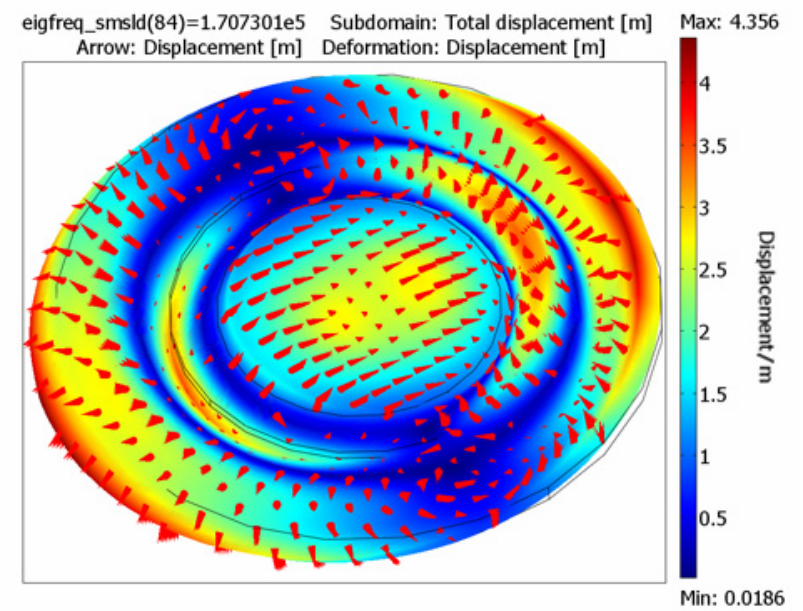

Fig. 5. Second harmonic of the diagonal FSM at $171 \mathrm{kHz}$ with slight vertical displacement in the center. 

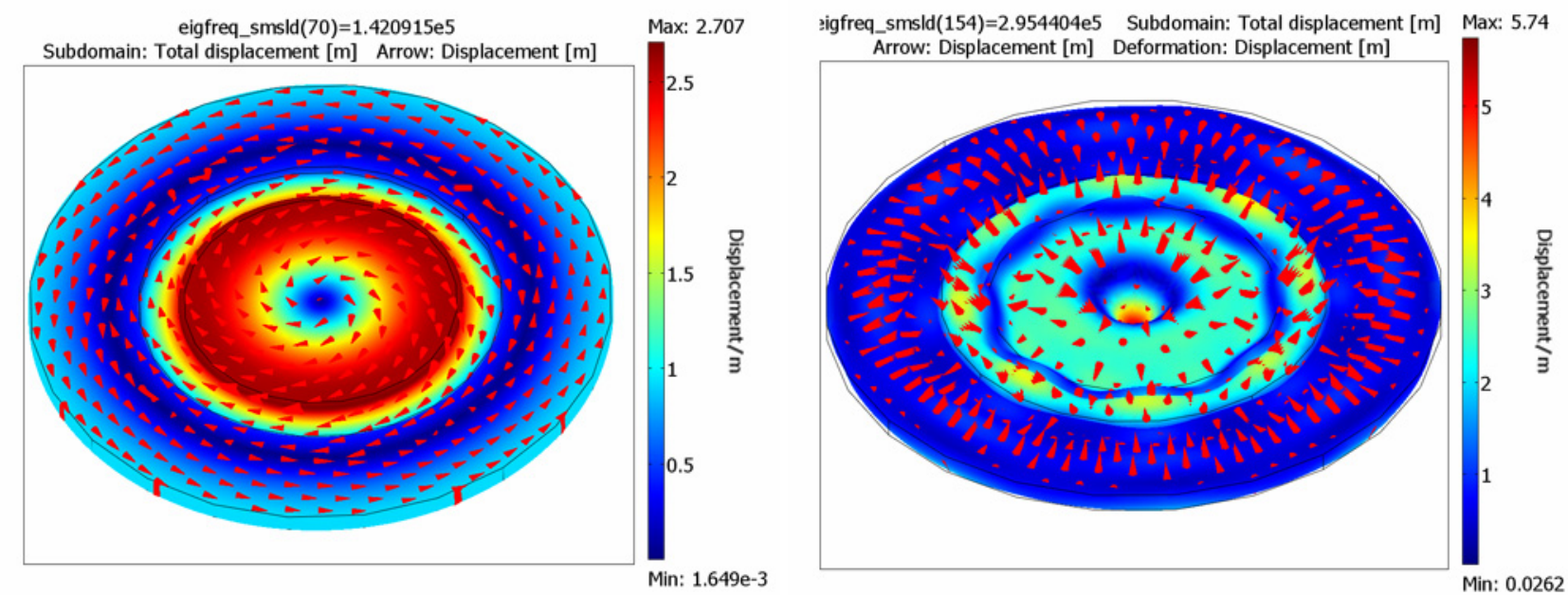

Fig. 6. Fundamental circular face shear mode at $142 \mathrm{kHz}$ with no out-of-plane component for liquid property sensing (left) and efficiently excited radial flexural plate mode at $295 \mathrm{kHz}$ for liquid volume sensing.

environment. The circular FSM shows no out-of-plane vibration both in the eigenmode and in the excitation analysis. The acoustic energy is well trapped in the central mesa structure. This behavior is ideal for sensing the liquid properties, such as viscosity and density, with shear waves. There is primarily only viscous damping of this resonance, additional damping due to destructive interference of compressional waves is minimal. The total volume and mass of the liquid on the resonator has no significant influence on the sensor response, and a direct proportional relationship between the density viscosity product and the frequency shift of the resonance can be established. These results have also been achieved by other groups, and similar conclusions could be drawn. Circular FSM or torsional plate resonators are suitable sensor devices for measuring liquid properties and distinguishing between different fluids [9].

The radial flexural plate mode on the other hand is dominated by out-of-plane components of the vibration. Due to the coupling with a radial shear movement, the inplane forces generated by a circular spiral coil are highly efficient at exciting this mode of vibration. The vertical movement radiates compressional waves into the liquid and is thus highly sensitive to the surface level height of the liquid. This device is suitable for measuring small samples of liquid volume with high precision. The influence of liquid property changes is negligible compared to the effect of the liquid height [10]. This simulation and the modeling have been employed for silicon transducers, aluminum and other metal elements, and polymer films. The anisotropy of some of these materials influences the possible mode shapes, but does not directly affect the excitation of the resonances.

To verify the simulated modes of vibration, we have recently started to characterize the different resonances using a Polytec Microsystem Analyzer, a laser vibrometer and stroboscope system for both out-of-plane and inplane measurements.

\section{Measurement results}

Our liquid phase experiments focused on the aluminum mesa-shaped resonator (inset Fig. 8) and the comparison of different modes. The results have proven to be as expected, with the circular and diagonal FSM achieving the best results in distinguishing between different liquids and measuring density and viscosity, while the flexural modes are most sensitive to the liquid volume.

Fig. 7 shows a fundamental resonance in air of a diagonal face shear mode at $217 \mathrm{kHz}$. A liquid dampens this resonance noticeably, however the frequency shift of a few $\mathrm{kHz}$ can still be measured. Better results can be achieved with the radial flexure resonance at $288 \mathrm{kHz}$ and the circular thickness shear mode at $1.5 \mathrm{MHz}$, each suitable for the abovementioned measurement modes (Fig. 8). We have focused on the most recent measurement results in [10].

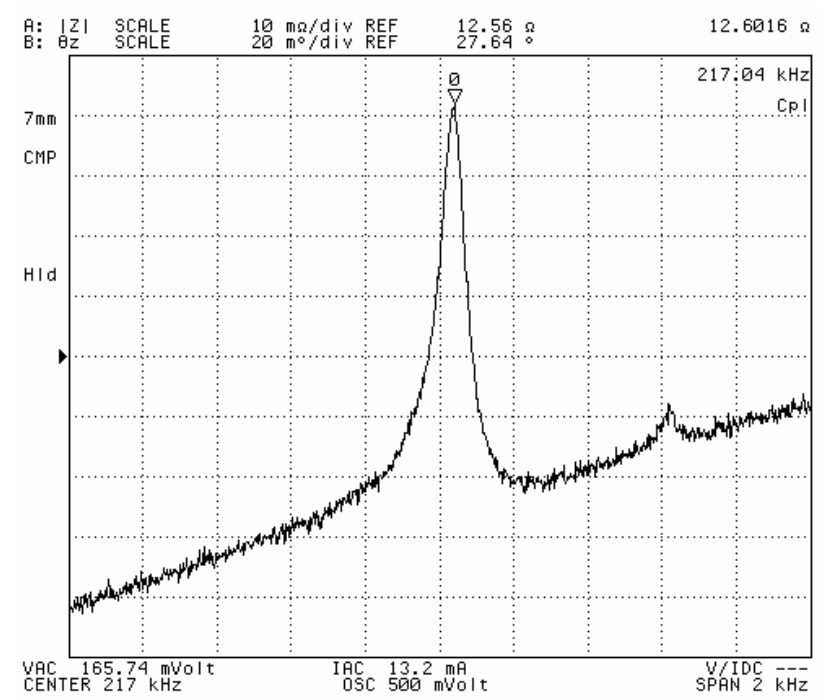

Fig. 7. Diagonal face shear mode in air at $217 \mathrm{kHz}$. 

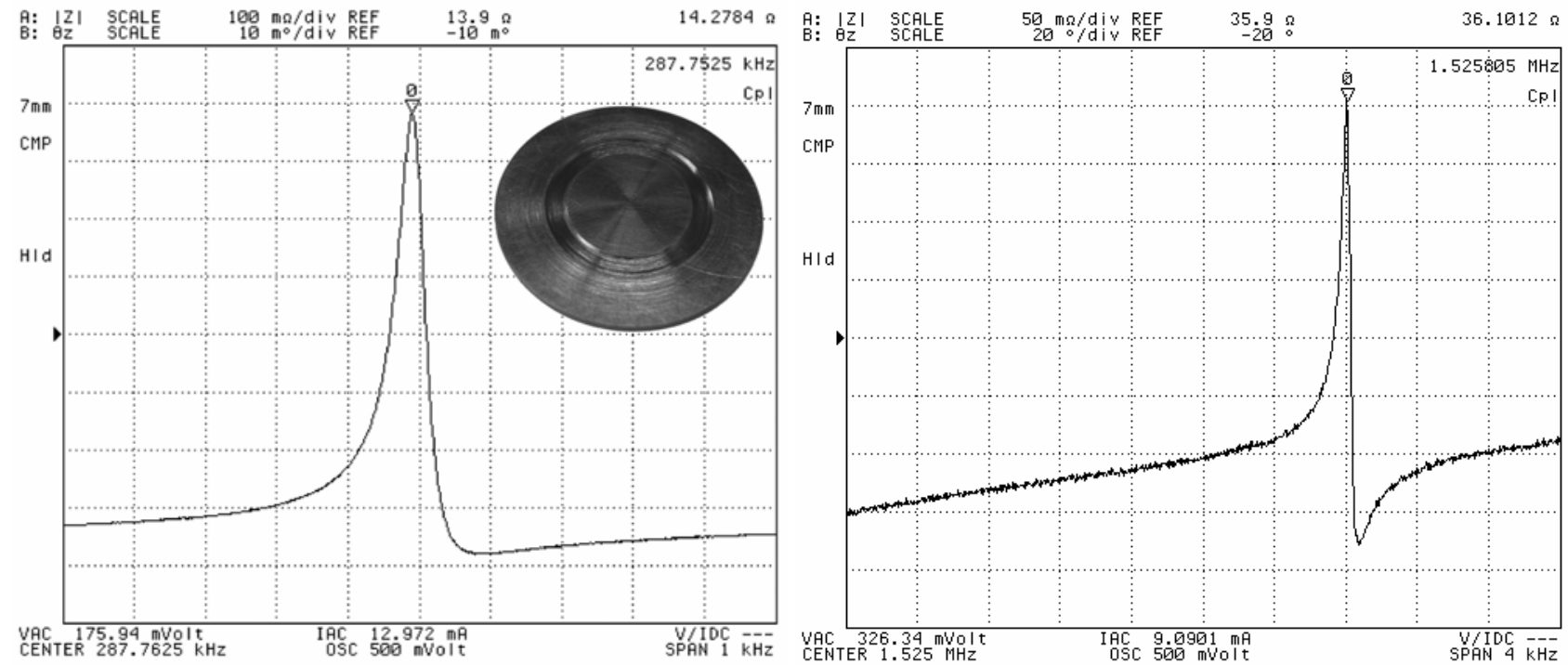

Fig. 8. Measured radial flexural plate resonance in air at $288 \mathrm{kHz}$ (left) of the aluminum resonator (inset) and circular thickness shear mode resonance in air at $1.5 \mathrm{MHz}$ (left) displaying similar behavior as the circular face shear mode at $142 \mathrm{kHz}$.

\section{Conclusions}

We have demonstrated the concept of electromagnetic excitation of acoustic resonator sensors for liquid phase sensing and discussed the different aspects of transducer and measurement setup design. In this contribution, we have focused on the most recent modeling and simulation of the current resonator elements. These elements, made of different materials like aluminum, brass, silicon, or polymer foil, all show suitable resonant behavior. Currently, the primary resonances are different face shear mode vibrations. Simulation and measurement agree on resonant frequency and mode shape for the specific transducer design. Therefore, it is possible to select promising simulated modes prior to measurement and predict the resonant behavior under different acoustic load conditions.

Current and future aspects of our research include the transfer of these results to thickness shear modes for increased sensitivity. Furthermore, we will design and fabricate new, miniaturized transducer elements, optimized for specific measurements. The utilization of low-cost silicon sensor arrays and polymer transducers allows for a wide range of possible applications.

\section{E. References}

[1] S.V. Krishnaswamy, J. Rosenbaum, S. Horwitz, C. Vale, R.A. Moore, "Film bulk acoustic wave resonator technology”, Proc. IEEE Ultrason. Symp., Honolulu, December 1990, pp. 529-536.
[2] X.C. Jin, I. Ladabaum, B.T. Khuri-Yakub, "The microfabrication of capacitive ultrasonic transducers," IEEE J. Microelectromech. Syst., vol. 7, pp. 295-302, 1998.

[3] J. Krautkrämer, H. Krautkrämer, Werkstoffprüfung mit Ultraschall, Berlin: Springer-Verlag, ch. 8, 1961.

[4] J.J. Quinn, "Electromagnetic generation of acoustic waves and the surface impedance of metals", Phys. Lett., vol. 25A, pp. 522-523, 1967.

[5] A.C. Stevenson, C.R. Lowe, "Magnetic-acousticresonator sensors (MARS): a new sensing methodology" Sensors Actuators A, vol. 72, pp. 32-37, 1999.

[6] F. Lucklum, P. Hauptmann, N.F. de Rooij, "Magnetic direct generation of acoustic resonances in silicon membranes”, Meas. Sci. Technol., vol. 17, pp. 719-726, 2006.

[7] F. Lucklum, B. Jakoby, P. Hauptmann, N.F. de Rooij, "Remote Electromagnetic Excitation of High-Q Silicon Resonator Sensors”, Proc. IEEE Int. Freq. Contr. Symp., Miami, June 2006, pp. 139-144.

[8] F. Lucklum, B. Jakoby, "Acoustic Wave Generation and Detection in Non-Piezoelectric High-Q Resonators”, Proc. IEEE Ultrason. Symp., Vancouver, October 2006, pp. 1132-1135.

[9] M.K. Kang, R. Huang, T. Knowles, "Energy-Trapping Torsional-Mode Resonators for Liquid Sensing”, Proc. IEEE Int. Freq. Contr. Symp., Miami, June 2006, pp. 133138.

[10] F. Lucklum, B. Jakoby, "Novel Magnetic-Acoustic Face Shear Mode Resonators for Liquid Property Sensing”, Digest Tech. Papers Transducers'07, Lyon, June 2007, to be published. 